\title{
Microwave ablation therapy for treating primary and secondary lung tumours: technical note
}

\section{La terapia ablativa con microonde nel trattamento delle lesioni tumorali primitive e secondarie del polmone: nota tecnica}

\author{
G. Carrafiello ${ }^{1} \cdot$ M. Mangini ${ }^{1} \cdot$ I. De Bernardi ${ }^{1} \cdot$ F. Fontana $^{1} \cdot$ G. Dionigi ${ }^{2} \cdot$ S. Cuffari $^{3}$ \\ A. Imperatori ${ }^{4} \cdot$ D. Laganà ${ }^{1} \cdot$ C. Fugazzola $^{1}$
}

\begin{abstract}
${ }^{1}$ Dipartimento di Radiologia, ${ }^{2}$ Dipartimento di Scienze Chirurgiche, ${ }^{3}$ Servizio di Anestesia, ${ }^{4}$ Chirurgia Toracica, Università degli Studi dell'Insubria, Ospedale di Circolo Varese, Viale L. Borri 57, 21100 Varese, Italy

Correspondence to: G. Carrafiello,Tel.: +39-0332-278763, Fax: +39-0332-261440, e-mail: gcarraf@tin.it
\end{abstract}

Received: 17 August 2009 / Accepted: 2 October 2009 / Published online: 29 March 2010

(C) Springer-Verlag 2010

\begin{abstract}
Purpose. The purpose of our study was to retrospectively evaluate the feasibility, safety and effectiveness of microwave ablation (MWA) in nine patients with unresectable lung tumour.

Materials and methods. Ten lesions were treated in ten ablation sessions in nine patients. The treatments were performed with a microwave generator with $45 \mathrm{~W}$ and 915 $\mathrm{MHz}$ connected to a 14.5-gauge antenna for $10 \mathrm{~min}$. Antenna placement was performed with computed tomography (CT) fluoroscopy guidance or XperGuide. All patients underwent CT follow-up at 1, 3 and 6 months from the procedure.

Results. Technical success was obtained in all cases; mortality at 30 days was $0 \%$.

Conclusions. This study shows that in selected patients, MWA is a valid alternative to other ablative techniques. Further studies are required to demonstrate the short- and long-term effects of this technique and to make a comparison with other available ablation systems, especially with radiofrequency.
\end{abstract}

Keywords Microwave ablation · Lung tumour

\section{Riassunto}

Obiettivo. Lo scopo dello studio è stato quello di valutare il successo tecnico, la sicurezza, l'efficacia della metodica del trattamento ablativo mediante microonde $(M W)$ in 9 pazienti affetti da neoplasia polmonare non trattabile chirurgicamente.

Materiali e metodi. Sono state trattate 10 masse polmonari in 10 sessioni d'ablazione in 9 pazienti. È stato utilizzato un sistema ablativo costituito da un generatore di MW a $45 \mathrm{~W}$ e $915 \mathrm{MHz}$ connesso ad un antenna 14,5 G, per un tempo di ablazione totale di 10 minuti. Il posizionamento dell'antenna è stato eseguito sotto guida fluoro-tomografia computerizzata (TC) o XperGuide. I pazienti sono stati sottoposti a follow-up mediante TC a 1, 3, 6 mesi dalla procedura.

Risultati. Si è ottenuto un successo tecnico in tutti i casi; il tasso di mortalità a 30 giorni è stato dello $0 \%$.

Conclusioni. Questo studio dimostra che, in pazienti selezionati, la termoablazione con microonde rappresenta una valida alternativa ad altre tecniche ablative. Sono tuttavia necessari ulteriori studi per confermare a breve e lungo termine l'efficacia di questa metodologia e permettere un confronto con altri sistemi ablativi, in particolare rispetto alla radiofrequenza.

Parole chiave Ablazione con microonde - Tumore polmonare 


\section{Introduction}

Surgery is still the treatment of choice for non-small-cell lung cancer (NSCLC) [1, 2]. However, only $20 \%$ of all diagnosed NSCLCs are resectable [1,2]. This has prompted the development of alternatives to surgical treatment, such as ablation techniques, to enable local control of unresectable tumours [3,4]. Over the years, various ablation techniques have been developed, including ethanol ablation, laser ablation, cryoablation and radiofrequency ablation (RFA) [3, 4]. The most widely used technique is radiofrequency ablation [5-12].

Microwave ablation (MWA) therapy is a relatively new technique that can be applied to different types of tumours. It is able to offer all the benefits of RFA as well as some substantial advantages. These include larger volumes of cellular necrosis, reduced procedure times, greater temperatures delivered to the target lesion, the possibility of the simultaneous use of multiple antennae, efficacy on lesions with cystic components and/or in proximity to vascular structures $>3 \mathrm{~mm}$ in diameter with a reduction in the heat-sink effect, and less intraprocedural pain $[5,6$, 13-22].

In the radiation spectrum, microwaves lie between infrared and radio waves, with a frequency between 900 and 2,450 MHz [6]. Microwaves induce the oscillation of water molecules at a speed between 2 and 5 billion times per second, depending on the frequency of the microwaves themselves $[5,6,16,17]$. Cell death is produced by thermocoagulation and necrosis as a result of the heat generated by oscillations of ions induced by the microwaves, the degree of which is proportional to the intensity and frequency of the microwaves themselves [6,7]. The aim of this study was to demonstrate the technical success, safety and efficacy of MWA in nine patients affected by unresectable lung cancer.

\section{Materials and methods}

Patients

This retrospective study was approved by the ethics committee of our centre. Nine patients (seven men, two women) with a mean age of 78 (range 69-88) years who underwent percutaneous MWA of ten intraparenchymal pulmonary masses (mean 1.1 lesions per patient) in ten ablation sessions between 26 November 2008 and 12 August 2009 were enrolled in the study (Table 1). All selected patients had neoplastic disease that was judged to be inoperable on the basis of tumour stage, comorbidities, advanced age and/or refusal to undergo surgery. Indication, risks and benefits of the procedure

\section{Introduzione}

La terapia chirurgica rimane il trattamento di scelta per il carcinoma polmonare non a piccole cellule (NSCLC) [1, 2]. Tuttavia, solo il $20 \%$ di tutti i NSCLC diagnosticati risulta essere chirurgicamente resecabile [1,2] Ciò ha pertanto spinto verso la ricerca di metodi alternativi al trattamento chirurgico, quali le tecniche ablative, per consentire il controllo locale di tumori non resecabili $[3,4]$. Nel corso degli anni sono state sviluppate diverse tecniche ablative: ablazione con etanolo, laserablazione, crioablazione $e$ termoablazione mediante radiofrequenza (RFA) [3, 4]. Attualmente la metodica più diffusamente impiegata nella pratica clinica è la radiofrequenza [5-12].

La tecnologia delle microonde $(M W)$ è un trattamento ablativo relativamente nuovo applicabile a differenti tipologie di neoplasie, in grado di offrire tutti $i$ benefici della radiofrequenza, presentando inoltre sostanziali vantaggi, quali: volumi di necrosi cellulare più ampi, riduzione del tempo di procedura, maggiori temperature alla lesione target, possibilità di utilizzare multiple antenne simultaneamente, efficacia su lesioni con componente cistica e/o in prossimità di strutture vascolari $>3 \mathrm{~mm}$ di diametro con riduzione dell'heat sink effect, minore dolore intraprocedurale [5, 6, 13-22].

Nello spettro delle radiazioni le microonde si collocano tra le radiazioni infrarosse e le radioonde con una frequenza tra 900 e $2450 \mathrm{MHz}$ [6]. Le microonde, inducendo l'oscillazione delle molecole d'acqua con movimenti di rotazione su se stesse in avanti ed indietro ad una velocità di 2-5 miliardi di volte al secondo dipendente dalla frequenza delle onde stesse [5, 6, 16, 17]. La morte cellulare avviene attraverso necrosi termocoagulativa grazie al calore generato dall'oscillazione degli ioni indotta dalle microonde in proporzione all'intensità ed alla frequenza delle onde stesse [6, 7]. Lo scopo dello studio è stato quello di dimostrarare il successo tecnico, la sicurezza, l'efficacia della metodica del trattamento ablativo mediante MW in 9 pazienti affetti da neoplasia polmonare non trattabile chirurgicamente.

\section{Materiali e metodi}

\section{Pazienti}

Questo studio retrospettivo è stato approvato dal Comitato Etico del nostro ospedale. Dal 26 novembre 2008 al 12 agosto 2009 sono stati arruolati nello studio 9 pazienti (7 maschi, 2 femmine) con un'età media di 78 anni (range 69-88 anni) e sottoposti a trattamento termoablativo percutaneo con $M W$ di 10 masse intraparenchimali polmonari (media di 1,1 lesioni per paziente) in 10 sessioni d'ablazione (Tabella 1). Tutti i pazienti selezionati presentavano malattia neoplastica giudicata inoperabile in base allo 
Table 1 Patients and lesion characteristics

\begin{tabular}{|c|c|c|c|c|c|c|c|c|}
\hline Patient no. & Age & Sex & Histology & Stage & $\begin{array}{l}\text { No. of } \\
\text { masses }\end{array}$ & $\begin{array}{l}\text { Long-axis } \\
\text { diameter } \\
(\mathrm{mm})\end{array}$ & Location & $\begin{array}{l}\text { Follow-up } \\
\text { (months) }\end{array}$ \\
\hline \multirow[t]{2}{*}{1} & 72 & $\mathrm{~F}$ & Squamous cell carcinoma & IV & $1^{\mathrm{a}}$ & 35 & $\begin{array}{l}\text { Apicoposterior segment } \\
\text { left upper lobe }\end{array}$ & 3 \\
\hline & & & & & $\begin{array}{l}2^{\mathrm{a}} \text { (post- } \\
\text { MWA } \\
\text { recurrent } \\
\text { lesion) }\end{array}$ & 45 & & $<1$ \\
\hline 2 & 82 & M & Squamous cell carcinoma & II & 1 & 20 & $\begin{array}{l}\text { Apical segment } \\
\text { of left lower lobe }\end{array}$ & 5 \\
\hline 3 & 77 & M & Adenocarcinoma & IV (M1) & 1 & 20 & Right lower lobe & 5 \\
\hline 4 & 78 & M & Squamous cell carcinoma & I & 1 & 69 & Right lower lobe & 9 \\
\hline 5 & 69 & M & Neuroendocrine carcinoma & IV (M1) & 1 & 14 & Right lower lobe & 3 deceased \\
\hline 6 & 79 & M & Adenocarcinoma & IV (M1) & $\begin{array}{l}1^{\mathrm{a}} \\
2^{\mathrm{a}}\end{array}$ & $\begin{array}{l}49 \\
16\end{array}$ & Right middle lobe & 3 \\
\hline 7 & 82 & M & Adenocarcinoma & I & 1 & 15 & Left upper lobe & 3 \\
\hline 8 & 88 & M & Squamous cell carcinoma & II & 1 & 32 & Right lower lobe & 3 \\
\hline 9 & 75 & $\mathrm{~F}$ & Squamous cell carcinoma & IV & 1 & 25 & Left upper lobe & 1 \\
\hline
\end{tabular}

$M$, male; $F$, female; $M W A$, microwave ablation

Tabella 1 Pazienti e caratteristiche delle lesioni tumorali

\begin{tabular}{|c|c|c|c|c|c|c|c|c|}
\hline $\begin{array}{l}\text { Numero } \\
\text { pazienti }\end{array}$ & Età & Ses & Istologia & Stadio & $\begin{array}{l}\text { Numero } \\
\text { masse }\end{array}$ & $\begin{array}{l}\text { Diametro } \\
\text { massimo } \\
\text { lesione }(\mathrm{mm})\end{array}$ & Sede & $\begin{array}{l}\text { Follow-up } \\
\text { (mesi) }\end{array}$ \\
\hline \multirow[t]{2}{*}{1} & 72 & $F$ & Carcinoma squamoso & $I V$ & $1^{a}$ & 35 & $\begin{array}{l}\text { Segmento apico-dorsale } \\
\text { lobo superiore sinistro }\end{array}$ & 3 \\
\hline & & & & & $\begin{array}{l}2^{a} \text { (recidiva } \\
\text { lesione } \\
\text { post- } M W A)\end{array}$ & 45 & & $<1$ \\
\hline 2 & 82 & $M$ & Carcinoma squamoso & $I I$ & 1 & 20 & $\begin{array}{l}\text { Segmento apicale lobo } \\
\text { inferiore sinistro }\end{array}$ & 5 \\
\hline 3 & 77 & $M$ & Adenocarcinoma & $I V(M 1)$ & 1 & 20 & Lobo inferiore destro & 5 \\
\hline 4 & 78 & $M$ & Carcinoma squamoso & $I$ & 1 & 69 & Lobo inferiore destro & 9 \\
\hline 5 & 69 & $M$ & Carcinoma neuroendocrino & $I V(M 1)$ & 1 & 14 & Lobo inferiore destro & 3 morte \\
\hline 6 & 79 & $M$ & Adenocarcinoma & $I V(M 1)$ & $\begin{array}{l}1^{a} \\
2^{a}\end{array}$ & $\begin{array}{l}49 \\
16\end{array}$ & Lobo medio destro & 3 \\
\hline 7 & 82 & $M$ & Adenocarcinoma & $I$ & 1 & 15 & Lobo superiore sinistro & 3 \\
\hline 8 & 88 & $M$ & Carcinoma squamoso & $I I$ & 1 & 32 & Lobo inferiore destro & 3 \\
\hline 9 & 75 & $F$ & Carcinoma squamoso & $I V$ & 1 & 25 & Lobo superiore sinistro & 1 \\
\hline
\end{tabular}

$M$, maschio; $F$, femmina; MWA, ablazione con microonde

were discussed with all patients prior to treatment. The coagulation profile was in the normal range for all patients. Patients undergoing anticoagulant and/or antiaggregant therapy interrupted treatment at least 7 days before the procedure, with the introduction of fractionated heparin when necessary. Written informed consent was obtained from all patients. stadio, alla presenza di comorbidità, all'età avanzata e/o rifiutavano l'intervento chirurgico. L'indicazione, i rischi ed i benefici della procedura sono stati discussi prima del trattamento con tutti i pazienti. La crasi ematica è risultata essere nel range di normalità in tutti i pazienti. I pazienti in terapia anticoagulante elo antiaggregante hanno sospeso il trattamento almeno 7 giorni prima della procedura, 
Baseline imaging

Pretreatment imaging consisted of a thoracic multidetectorrow computed tomography (MDCT) scan extending to the abdomen (Aquilion 64, Toshiba, Japan) with and without contrast administration. Each MDCT scan was acquired with a thickness of $0.5 \mathrm{~mm}$, voltage of $120 \mathrm{kV}$ and tube current of $250 \mathrm{~mA}$. The contrast-enhanced scans were acquired after injection of $100 \mathrm{ml}$ of iodinated contrast agent (Visipaque 320 , GE Healthcare, USA) at an injection rate of $3 \mathrm{ml} / \mathrm{s}$, followed by injection of $40 \mathrm{ml}$ saline at a rate of $2 \mathrm{ml} / \mathrm{s}$. One week prior to the ablation treatment, all patients underwent percutaneous pulmonary biopsy under CT guidance, with histological confirmation of NSCLC for all lesions.

Pretreatment procedure

Before the beginning of each procedure, local anaesthesia of the antenna entrance site was achieved with subcutaneous injection of a 10-ml solution of $2 \%$ Carbocaine. Each patient was kept in a state of moderate sedation through intravenous administration of a combination of midazolam $(0.07-0.08 \mathrm{mg} / \mathrm{kg})$, propofol $(0.5-2.0 \mathrm{mg} / \mathrm{kg} / \mathrm{h})$ and fentanyl $(1-2 \mu \mathrm{g} / \mathrm{kg}$ ). Heart rate, electrocardiographic trace, oxygen saturation, respiratory frequency and blood pressure were continuously monitored throughout the procedure. During the entire ablation session, patients were monitored by an anaesthetist. Adequate antibiotic prophylaxis was achieved with intravenous administration of $1 \mathrm{~g}$ of cefazolin sodium (Ancef, SmithKline Beecham Pharmaceuticals, Philadelphia, USA) given every $8 \mathrm{~h}$ for $24 \mathrm{~h}$, beginning shortly before the procedure.

\section{Equipment}

\section{Imaging guidance}

In eight out of ten sessions (7/10 lesions), the antenna was placed under CT fluoroscopy guidance, whereas in the remaining two sessions (3/10 lesions), the procedure was performed with XperGuide. The choice of imaging guidance was made on a random basis.

Microwave equipment and percutaneous microwave ablation procedure

An ablation system was used comprising a microwave generator (Evident Microwave Ablation System, Covidien Ltd) capable of producing $45 \mathrm{~W}$ at $915 \mathrm{MHz}$, connected by coaxial cable to a 14.5-gauge straight microwave antenna with a $3.7-\mathrm{cm}$ radiating section. The antennae were continuously perfused with saline solution at room temperature at $60 \mathrm{ml} / \mathrm{min}$ to avoid possible thermal damage along the prox- introducendo eparina frazionata se necessario. Un consenso informato scritto è stato ottenuto per tutti i pazienti.

Baseline imaging

L'imaging pre-trattamento è stato ottenuto mediante indagine con tomografia computerizzata (TC) multistrato toracica con estensione addominale (Aquilion 64, Toshiba, Giappone), con e senza iniezione di mezzo di contrasto $(M d C)$. Ciascuna scansione TC è stata acquisita ad uno spessore di 0,5 mm, a $120 \mathrm{kV}$ e $250 \mathrm{~mA}$. Le scansioni con $M d C$ sono state eseguite iniettando $100 \mathrm{ml}$ di contrasto iodato (Visipaque 320, GE Healthcare, USA) ad una velocità di $3 \mathrm{ml} / \mathrm{s}$ seguito dall'iniezione di $40 \mathrm{ml}$ di soluzione salina ad una velocità di $2 \mathrm{ml} / \mathrm{s}$. Una settimana prima del trattamento ablativo tutti i pazienti sono stati sottoposti a biopsia polmonare percutanea sotto guida TC con conferma istologica di NSCLC per tutte le lesioni.

Procedure pre-trattamento

Prima dell'inizio di ciascuna procedura è stata eseguita un'anestesia locale in sede d'ingresso dell'antenna attraverso l'iniezione sottocutanea di una soluzione di $10 \mathrm{ml}$ al $2 \%$ di Carbocaina. Ciascun paziente è stato mantenuto in regime di sedazione moderata, ottenuta mediante la somministrazione per via endovenosa di una combinazione di midazolam (0,07-0,08 $\mathrm{mg} / \mathrm{kg})$, propofol $(0,5-2 \mathrm{mg} / \mathrm{kg} / \mathrm{h}) e$ fentanil $(1-2 \mu \mathrm{g} / \mathrm{kg})$. Durante il trattamento sono stati monitorati in continuo la frequenza cardiaca, il tracciato elettrocardiografico, la saturazione dell'ossigeno, la frequenza respiratoria, la pressione sanguigna. I pazienti, per tutta la sessione di ablazione, sono stati sorvegliati da un medico anestesita. Un'adeguata profilassi antibiotica ̀̀ stata raggiunta mediante la somministrazione endovena di $1 \mathrm{~g}$ di cefazolina sodica (Ancef, SmithKline Beecham Pharmaceuticals, Philadelphia, USA) somministrata ogni 8 ore per 24 ore, iniziando poco prima della procedura.

Equipaggiamento

Imaging guidance

In 8 sessioni su 10 (7/10 lesioni) il posizionamento dell'antenna è stato eseguito sotto guida fluoro-TC, mentre nelle restanti due sessioni (3/10 lesioni) la procedura è stata eseguita sotto guida XperGuide. La scelta della guida è stata presa secondo criterio casuale.

Apparecchiatura per microonde e procedura ablativa percutanea mediante microonde

È stato utilizzato un sistema ablativo costituito da un 
imal semiaxis of the antenna. According to manufacturer specifications, ablation was performed by inserting the antenna within the lesion and maintaining a power of $45 \mathrm{~W}$ for a total ablation time of $10 \mathrm{~min}$ in order to obtain a necrosis volume of approximately $3.5 \mathrm{~cm}$ in diameter (Table 2). Lesions with a maximum diameter $\leq 3 \mathrm{~cm}$ (8/10 lesions) generatore di microonde (Evident TM Microwave Ablation System, Covidien Ltd), in grado di produrre una potenza massima di $45 \mathrm{~W}$ ad una frequenza di $915 \mathrm{MHz}$, connesso, attraverso un cavo coassiale, ad un'antenna per microonde diritta e sottile 14,5 gauge e con sezione radiante di 3,7 cm. Le antenne sono state perfuse in continuo con soluzione

Table 2 Characteristics of the microwave ablation(MWA) procedure

\begin{tabular}{|c|c|c|c|c|c|c|c|c|}
\hline $\begin{array}{l}\text { No.- } \\
\text { Age-Sex }\end{array}$ & $\begin{array}{l}\text { No. of } \\
\text { masses }\end{array}$ & $\begin{array}{l}\text { Long-axis } \\
\text { diameter } \\
(\mathrm{mm})\end{array}$ & Location & $\begin{array}{l}\text { No. } \\
\text { antennae }\end{array}$ & $\begin{array}{l}\text { MWA } \\
\text { time } \\
(\min )\end{array}$ & $\begin{array}{l}\text { Frequency } \\
(\mathrm{MHz})\end{array}$ & Guidance & $\begin{array}{l}\text { Ablation } \\
\text { (complete/ } \\
\text { partial) }\end{array}$ \\
\hline \multirow[t]{2}{*}{$1-72-\mathrm{F}$} & $1^{\mathrm{a}}$ & 35 & $\begin{array}{l}\text { Apicoposterior segment } \\
\text { of left upper lobe }\end{array}$ & 1 & 10 & 45 & CT fluoroscopy & Complete \\
\hline & $\begin{array}{l}2^{\mathrm{a}} \text { (post- } \\
\text { MWA } \\
\text { recurrent } \\
\text { lesion) }\end{array}$ & 45 & & 1 & & & & \\
\hline 2-82-M & 1 & 20 & $\begin{array}{l}\text { Apical segment of left } \\
\text { lower lobe }\end{array}$ & 1 & 10 & 45 & XperGuide & Complete \\
\hline 3-77-M & 1 & 20 & Right lower lobe & 1 & 10 & 45 & CT fluoroscopy & Complete \\
\hline 4-78-M & 1 & 69 & Right lower lobe & 2 & 10 & 45 & CT fluoroscopy & Partial \\
\hline 5-69-M & 1 & 14 & Right lower lobe & 1 & 10 & 45 & CT fluoroscopy & Complete \\
\hline 6-79-M & $\begin{array}{l}1^{\mathrm{a}} \\
2^{\mathrm{a}}\end{array}$ & $\begin{array}{l}49 \\
16\end{array}$ & Right middle lobe & $\begin{array}{l}2 \\
1\end{array}$ & 10 & 45 & XperGuide & Complete \\
\hline 7-82-M & 1 & 15 & Left upper lobe & 1 & 10 & 45 & CT fluoroscopy & Complete \\
\hline 8-88-M & 1 & 32 & Right lower lobe & 1 & 10 & 45 & CT fluoroscopy & Complete \\
\hline $9-75-\mathrm{F}$ & 1 & 25 & Left upper lobe & 1 & 10 & 45 & CT fluoroscopy & Complete \\
\hline
\end{tabular}

$M$, male; $F$, female; $M W A$, microware ablation

Tabella 2 Caratteristiche della procedura ablativa con microonde

\begin{tabular}{|c|c|c|c|c|c|c|c|c|}
\hline $\begin{array}{l}\text { Numero- } \\
\text { età-sesso }\end{array}$ & $\begin{array}{l}\text { Numero } \\
\text { masse }\end{array}$ & $\begin{array}{l}\text { Dimensioni } \\
\text { lesioni } \\
(\mathrm{mm})\end{array}$ & Sede & $\begin{array}{l}\text { Numero } \\
\text { antenne }\end{array}$ & $\begin{array}{l}M W A \\
\text { tempo } \\
\text { (min) }\end{array}$ & $\begin{array}{l}\text { Potenza } \\
(\mathrm{MHz})\end{array}$ & Guida & $\begin{array}{l}\text { Ablazione } \\
\text { (completal } \\
\text { parziale) }\end{array}$ \\
\hline \multirow[t]{2}{*}{$1-72-F$} & $1^{a}$ & 35 & $\begin{array}{l}\text { Segmento apico-dorsale } \\
\text { lobo superiore sinistro }\end{array}$ & 1 & 10 & 45 & Fluoro-TC & Completa \\
\hline & $\begin{array}{l}2^{a} \\
\text { (recidiva } \\
\text { lesione } \\
\text { post- } \\
\text { mwa) }\end{array}$ & 45 & & 1 & & & & \\
\hline $2-82-M$ & 1 & 20 & $\begin{array}{l}\text { Segmento apicale lobo } \\
\text { inferiore sinistro }\end{array}$ & 1 & 10 & 45 & XperGuide & Completa \\
\hline $3-77-M$ & 1 & 20 & Lobo inferiore destro & 1 & 10 & 45 & Fluoro-TC & Completa \\
\hline $4-78-M$ & 1 & 69 & Lobo inferiore destro & 2 & 10 & 45 & Fluoro-TC & Parziale \\
\hline $5-69-M$ & 1 & 14 & Lobo inferiore destro & 1 & 10 & 45 & Fluoro-TC & Completa \\
\hline $6-79-M$ & $\begin{array}{l}1^{a} \\
2^{a}\end{array}$ & 16 & Lobo medio destro & $\begin{array}{l}2 \\
1\end{array}$ & 10 & 45 & XperGuide & Completa \\
\hline $7-82-M$ & 1 & 15 & Lobo superiore sinistro & 1 & 10 & 45 & Fluoro-TC & Completa \\
\hline $8-88-M$ & 1 & 32 & Lobo inferiore destro & 1 & 10 & 45 & Fluoro-TC & Completa \\
\hline $9-75-F$ & 1 & 25 & Lobo superiore sinistro & 1 & 10 & 45 & Fluoro-TC & Completa \\
\hline
\end{tabular}

$M$, maschio; $F$, femmina; MWA, ablazione con microonde 
were treated with a single antenna, whereas in lesions with a maximum diameter $>3 \mathrm{~cm}$ (2/10 lesions) two antennae were simultaneously positioned at a distance of $1 \mathrm{~cm}$ from each other to achieve adequate necrosis. Upon completion of the procedure, whether performed with CT fluoroscopic guidance or XperGuide, technical success was checked with CT fluoroscopy. After ablation, patients were transferred to the radiology recovery room for observation. At $2 \mathrm{~h}$ from the procedure, each patient underwent chest radiography to evaluate the presence of immediate complications.

Follow-up

All patients underwent CT follow-up with and without contrast administration at 1, 3 and 6 months in combination with complete blood and metabolic tests. All CT scans were acquired with $0.5-\mathrm{mm}$ thickness, $120 \mathrm{kV}$ and $250 \mathrm{~mA}$. The contrast-enhanced scans were obtained after administration of $100 \mathrm{ml}$ of iodinated contrast agent (Visipaque 320, GE Healthcare) injected at a rate of $3 \mathrm{ml} / \mathrm{s}$, followed by $40 \mathrm{ml}$ of saline solution at a rate of $2 \mathrm{ml} / \mathrm{s}$. The following imaging characteristics were evaluated: maximum postablation diameter of the mass; presence of cavitation; absent lesion enhancement ( $<15 \mathrm{HU}$ ); pleural effusion; adenopathy (short axis $>1 \mathrm{~cm}$ ); total volume of the ablated area (calculated with volume analysis software, Vitrea 2, software 3.8, Vital Images Inc, Minnesota, USA).

Outcomes: technical success, safety and efficacy of the technique

Technical success was defined as the correct positioning of the antennae within the lesion. Safety was defined as the frequency of intra-, peri- and postprocedural complications [6]. All complications were recorded and classified as major or minor in accordance with the classification system of the Society of Interventional Radiology [23]. All patients were evaluated for the presence of postablation syndrome [24]. Pain was evaluated on the basis of a visual analogue scale (VAS) [25]. The effectiveness of the technique was defined as complete absence of enhancement $(\mathrm{HU}<15)$ within the ablation zone on contrast-enhanced CT images [13].

\section{Results}

Tumours were measured in the three dimensions obtaining a mean index of the maximum diameter of $29.5 \mathrm{~mm}$ (range 14-69 mm) (Table 3). In five cases, the masses were located in the right lung, with the remaining cases in the left lung. Histological analysis showed that all lesions were NSCLC: six squamous-cell pulmonary carcinomas, three adenocarcinomas and one neuroendocrine carcinoma (Table 1). In two patients, fisiologia a temperatura ambiente ad una velocità di 60 $\mathrm{ml} / \mathrm{min}$ al fine di prevenire eventuali danni termici lungo il semiasse prossimale dell'antenna. Come suggerito dalla casa costruttrice, il trattamento ablativo è stato eseguito inserendo l'antenna all'interno della lesione, mantendendo una potenza di 45 W per un tempo di ablazione totale di 10 minuti, al fine di ottenere un volume di necrosi approssimativo di 3,5 cm di diametro (Tabella 2). Le lesioni con diametro massimo $\leq 3 \mathrm{~cm}$ (8/10 lesioni) sono state trattate con singola antenna, mentre nel caso di lesioni con diametro massimo $>3 \mathrm{~cm}$ (2/10 lesioni) sono state posizionate due antenne simultaneamente ad una distanza di circa $1 \mathrm{~cm}$ l'una dall'altra per ottenere un'adeguata necrosi. Al termine di ogni procedura, eseguita sia sotto guida fluoroTC che XperGuide, è stato eseguito un controllo fluoro-TC. Dopo l'ablazione i pazienti sono stati trasferiti presso la recovery room radiologica in osservazione. Ciascun paziente è stato sottoposto a 2 ore dalla procedura ad una radiografia del torace al fine di valutare l'eventuale presenza di complicanze immediate.

Follow-up

I pazienti sono stati sottoposti a follow-up mediante TC senza e con iniezione di MdC ad 1, 3 e 6 mesi dalla procedura in associazione ad indagini sieroematiche e metaboliche complete. Tutte le scansioni TC sono state acquisite con spessore di 0,5 mm, a $120 \mathrm{kV}$ e $250 \mathrm{~mA}$. Le scansioni con MdC sono state eseguite iniettando $100 \mathrm{ml}$ di contrasto iodato (Visipaque 320, GE Healthcare) ad una velocità di 3 $\mathrm{ml} / \mathrm{s}$ seguito dall'iniezione di $40 \mathrm{ml}$ di soluzione salina ad una velocità di $2 \mathrm{ml} / \mathrm{s}$. Sono state valutate le seguenti caratteristiche all'imaging: diametro massimo post-ablazione della massa; presenza di cavitazione; assenza di ehnancement della lesione (<15 H); soffusione pleurica; adenopatia (asse corto $>1 \mathrm{~cm}$ ), volume totale dell'area ablata (calcolato mediante l'utilizzo di un software, Vitrea 2, software 3.8, Vital Images Inc, Minnesota, USA).

Outcomes: successo tecnico, sicurezza ed efficacia della metodica

Il successo tecnico è stato definito come il corretto posizionamento dell'antenna all'interno dell lesione. La sicurezza è stata definita come la frequenza di complicanze intra-, peri- $e$ post-procedurali [6]. Tutte le complicanze sono state registrate e classificate in maggiori e minori in base alla classificazione SIR [23]. Tutti i pazienti sono stati indagati in merito alla sindrome post-ablazione [24]. Il dolore è stato valutato in base a scale analogiche visive (visual analog scale [VAS] score) [25]. L'efficacia della metodica è stata definita come la completa assenza di ehnancement $(H<15)$ all'interno della zona ablata alle scansioni TC con iniezioni di MdC [13]. 
the tumours were metastases from primary pulmonary tumours located in the contralateral lung (stage IV, M1).

\section{Technical success}

Technical success was $100 \%$. In all cases, the antenna was correctly placed within the lesion [6].

\section{Safety}

No major complications were recorded in the intra- and periprocedural period. The 30-day postablation mortality rate was $0 \%$. There was one death at 3 months of a 69 -year-old male patient affected by stage IV neuroendocrine carcinoma of the left lobe caused by a combination of factors related to the systemic disease associated with comorbidities. The death was therefore only relatively related to the ablation therapy. Ablation therapy nonetheless produced complete necrosis of the mass in this patient. Asymptomatic grade 1 pneumothorax was recorded in three patients. There were no cases of symptomatic grade 2 pneumothorax, nor were there any cases of skin burns induced by microwave-related thermal damage $[12,21]$. No patient was diagnosed with a postablation syndrome, had a VAS score indicating a need for pain relief, or patient required admission to an intensive care unit.

\section{Efficacy}

Efficacy $90 \%$. Complete necrosis was observed in nine out of ten lesions.

Imaging characteristics of lesions during follow-up

CT fluoroscopy scans obtained in the periprocedural period showed the presence of tumour necrosis as a result of the thermal effect induced by the microwaves. A common finding was areas of ground-glass attenuation within the ablated lesion and extending to the areas around the antenna. Additional CT scans were performed at intervals of 1 (all patients), 3 (8/9 patients) and 6 months (3/9 patients). After an initial increase of $0.67 \mathrm{~cm}$ in maximum diameters, there was a persistent reduction in diameter of the ablated areas at subsequent examinations, consistent with consolidation of the pulmonary parenchyma. During follow-up imaging, the presence of a cavitation was noted in $50 \%$ of cases (5/10 lesions) (Fig. 1). Minor pleural effusions were found in $20 \%$ of cases (2/10 lesions). Adenopathies with maximum dimensions $<1 \mathrm{~cm}$ were found at the level of the treated lesion in $10 \%$ of cases (1/10 lesions).

Disease recurrence was detected at 3 months in one case. The patient underwent additional ablation treatment at 3 months from initial treatment, producing complete necrosis of the recurrent lesion (Fig. 2), which was evaluated by CT

\section{Risultati}

Le lesioni tumorali sono state misurate nelle tre dimensioni ottendendo un indice medio del diametro massimo di 29,5 mm (range 14-69 mm) (Tabella 3). In 5 casi le masse tumorali erano situate a livello del polmone destro, nei restanti casi a livello del polmone sinistro. Secondo l'analisi istologica tutte le lesioni considerate sono risultate essere neoplasie polmonari NSCLC: 6 carcinomi polmonari a cellule squamose, 3 adenocarcinomi, 1 carcinoma neuroendocrino (Tabella 1). In 2 pazienti le lesioni sono risultate essere metastasi di tumori primitivi polmonari a livello del polmone controlaterale (stadio IV, MI).

Successo tecnico

Il successo tecnico è stato del 100\%: in tutti i casi l'antenna è stata correttamente posizionata all'interno della lesione [6].

\section{Sicurezza}

Non abbiamo riportato complicanze maggiori nel periodo intra-e peri-procedurale. Il tasso di mortalità a 30 giorni post-ablazione è stato dello $0 \%$. A 3 mesi dal trattamento si è verificata morte in un paziente maschio di 69 anni, affetto da carcinoma neuroendocrino stadio IV del lobo sinistro, causata dalla combinazione di fattori legati alla patologia sistemica in associazione a comorbidità. La morte è risultata essere solo relativamente connessa al trattamento ablativo. Il trattamento ablativo ha comunque permesso una necrosi completa della massa. In 3 pazienti si è verificato pneumotorace di grado I/asintomatico. Non c'è stato alcun riscontro di pneumotoraci di grado II/sintomatico. Non si sono verificate ustioni cutanee indotte dal danno termico microonde-correlato [12, 21]. Tutti i pazienti sono stati indagati in merito alla sindrome post-ablazione [23]. In questo studio in nessun paziente è stata diagnosticata sindrome post-ablazione. Nessun paziente ha espresso un valore, secondo il VAS score, tale da richiedere terapia antalgica. Nessun paziente ha necessitato il ricovero presso l'unità operativa di terapia intensiva.

Efficacia della metodica

L'efficacia della metodica è stata del 90\%: è stata osservata una necrosi completa in 9 lesioni su 10.

Caratteristiche all'imaging delle lesioni durante il follow-up

Le scansioni fluoro-TC eseguite nell'immediato periodo peri-procedurale hanno dimostrato la presenza di necrosi 
Table 3 Lesion characteristics at computed tomography (CT) follow-up

\begin{tabular}{|c|c|c|c|c|c|c|c|c|}
\hline $\begin{array}{l}\text { No.- } \\
\text { Age-Sex }\end{array}$ & $\begin{array}{l}\text { No. of } \\
\text { masses }\end{array}$ & $\begin{array}{l}\text { Pre-MWA } \\
\text { long-axis } \\
\text { diameter } \\
(\mathrm{mm})\end{array}$ & $\begin{array}{l}\text { Long-axis } \\
\text { diameter at } \\
1 \text { month } \\
(\mathrm{mm})\end{array}$ & $\begin{array}{l}\text { Long-axis } \\
\text { diameter at } \\
3 \text { months } \\
(\mathrm{mm})\end{array}$ & $\begin{array}{l}\text { Contrast } \\
\text { enhancement } \\
(\mathrm{HU})\end{array}$ & $\begin{array}{l}\text { Cavitation } \\
\text { effusion }\end{array}$ & Pleural & Adenopathy \\
\hline \multirow[t]{2}{*}{$1-72-\mathrm{F}$} & $1^{\mathrm{a}}$ & 35 & 45 & 45 & 40 & Absent & Absent & Absent \\
\hline & $2^{\mathrm{a}}$ recurrent & 45 & 47 & - & $<15$ & Absent & Absent & Absent \\
\hline 2-82-M & 1 & 20 & 24 & 21 & $<15$ & Present & Absent & $<1 \mathrm{~cm}$ \\
\hline 3-77-M & 1 & 20 & 18 & 18 & $<15$ & Present & Present & Absent \\
\hline 4-78-M & 1 & 69 & 75 & 68 & $<15$ & Present & Present & Absent \\
\hline 5-69-M & 1 & 14 & 24 & $\begin{array}{l}\text { Patient death } \\
\text { at } 3 \text { months } \\
\text { after MWA }\end{array}$ & $<15$ & Absent & Absent & Absent \\
\hline \multirow[t]{2}{*}{ 6-79-M } & $1^{\mathrm{a}}$ & 49 & 52 & 45 & $<15$ & Absent & Absent & Absent \\
\hline & $2^{a}$ & 16 & 23 & 45 & $<15$ & Absent & Absent & Absent \\
\hline 7-82-M & 1 & 15 & 35 & 20 & $<15$ & Present & Absent & Absent \\
\hline 8-88-M & 1 & 32 & 40 & 35 & $<15$ & Absent & Absent & Absent \\
\hline $9-75-\mathrm{F}$ & 1 & 25 & 32 & - & $<15$ & Present & Absent & Absent \\
\hline
\end{tabular}

$M$, male; $F$, female; $M W A$, microwave ablation; $F U$, follow-up

Tabella 3 Caratteristiche delle lesioni al follow-up mediante TC

\begin{tabular}{|c|c|c|c|c|c|c|c|c|}
\hline $\begin{array}{l}\text { Numero- } \\
\text { età-sesso }\end{array}$ & $\begin{array}{l}\text { Numero } \\
\text { masse }\end{array}$ & $\begin{array}{l}\text { Dimensioni } \\
\text { lesioni pre- } \\
\text { MWA ( } \mathrm{mm})\end{array}$ & $\begin{array}{l}\text { Dimensioni } \\
\text { lesioni } F U \\
1 \text { mese }(\mathrm{mm})\end{array}$ & $\begin{array}{l}\text { Dimensioni } \\
\text { lesioni FU } \\
3 \text { mesi }(\mathrm{mm})\end{array}$ & $\begin{array}{l}\text { Contrast } \\
\text { ehnancement } \\
(H)\end{array}$ & Cavitazione & $\begin{array}{l}\text { Soffusione } \\
\text { pleurica }\end{array}$ & $\begin{array}{l}\text { Linfoade- } \\
\text { nopatia }\end{array}$ \\
\hline $1-72-F$ & $\begin{array}{l}1^{a} \\
2^{a} \text { recidiva }\end{array}$ & $\begin{array}{l}35 \\
45\end{array}$ & $\begin{array}{l}45 \\
47\end{array}$ & $\begin{array}{l}45 \\
-\end{array}$ & $\begin{array}{l}40 \\
<15\end{array}$ & $\begin{array}{l}\text { Assente } \\
\text { Assente }\end{array}$ & $\begin{array}{l}\text { Assente } \\
\text { Assente }\end{array}$ & $\begin{array}{l}\text { Assente } \\
\text { Assente }\end{array}$ \\
\hline $2-82-M$ & 1 & 20 & 24 & 21 & $<15$ & Presente & Assente & $<1 \mathrm{~cm}$ \\
\hline $3-77-M$ & 1 & 20 & 18 & 18 & $<15$ & Presente & Presente & Assente \\
\hline $4-78-M$ & 1 & 69 & 75 & 68 & $<15$ & Presente & Presente & Assente \\
\hline $5-69-M$ & 1 & 14 & 24 & $\begin{array}{l}\text { Morte paziente } \\
\text { a } 3 \text { mesi } \\
\text { da MWA }\end{array}$ & $<15$ & Assente & Assente & Assente \\
\hline 6-79-M & $\begin{array}{l}1^{a} \\
2^{a}\end{array}$ & $\begin{array}{l}49 \\
16\end{array}$ & $\begin{array}{l}52 \\
23\end{array}$ & $\begin{array}{l}45 \\
45\end{array}$ & $\begin{array}{l}<15 \\
<15\end{array}$ & $\begin{array}{l}\text { Assente } \\
\text { Assente }\end{array}$ & $\begin{array}{l}\text { Assente } \\
\text { Assente }\end{array}$ & $\begin{array}{l}\text { Assente } \\
\text { Assente }\end{array}$ \\
\hline 7-82-M & 1 & 15 & 35 & 20 & $<15$ & Presente & Assente & Assente \\
\hline 8-88-M & 1 & 32 & 40 & 35 & $<15$ & Assente & Assente & Assente \\
\hline $9-75-F$ & 1 & 25 & 32 & - & $<15$ & Presente & Assente & Assente \\
\hline
\end{tabular}

$M$, maschio; $F$, femmina; MWA, ablazione con microonde; $F U$, follow-up

several days after the procedure. The total follow-up period was recorded in all patients as the time from the date of the procedure to the most recent CT scan, with mean duration being 3.6 (range 1-9) months.

\section{Discussion}

Over the years, several ablation techniques have been developed for local control of unresectable tumours: ethanol ablation, laser ablation, cryoablation and radiofrequency ther- della massa tumorale come effetto termico indotto dalle microonde. Di comune riscontro è stata l'osservazione di aree soffuse di opacificazione a vetro smerigliato all'interno della lesione ablata ed estese alle aree attorno l'antenna. Ad intervalli di 1 (tutti i pazienti), 3 (8/9 pazienti) e 6 mesi (3/9 pazienti) sono state eseguite ulteriori scansioni TC. È stato quindi osservato un incremento iniziale dei diametri massimi nel periodo peri-procedurale, con un aumento del diametro massimo di 0,67 cm. Ai successivi controlli si è notato una persistente riduzione del diametro dell'area ablata, conforme al consolidamento del 

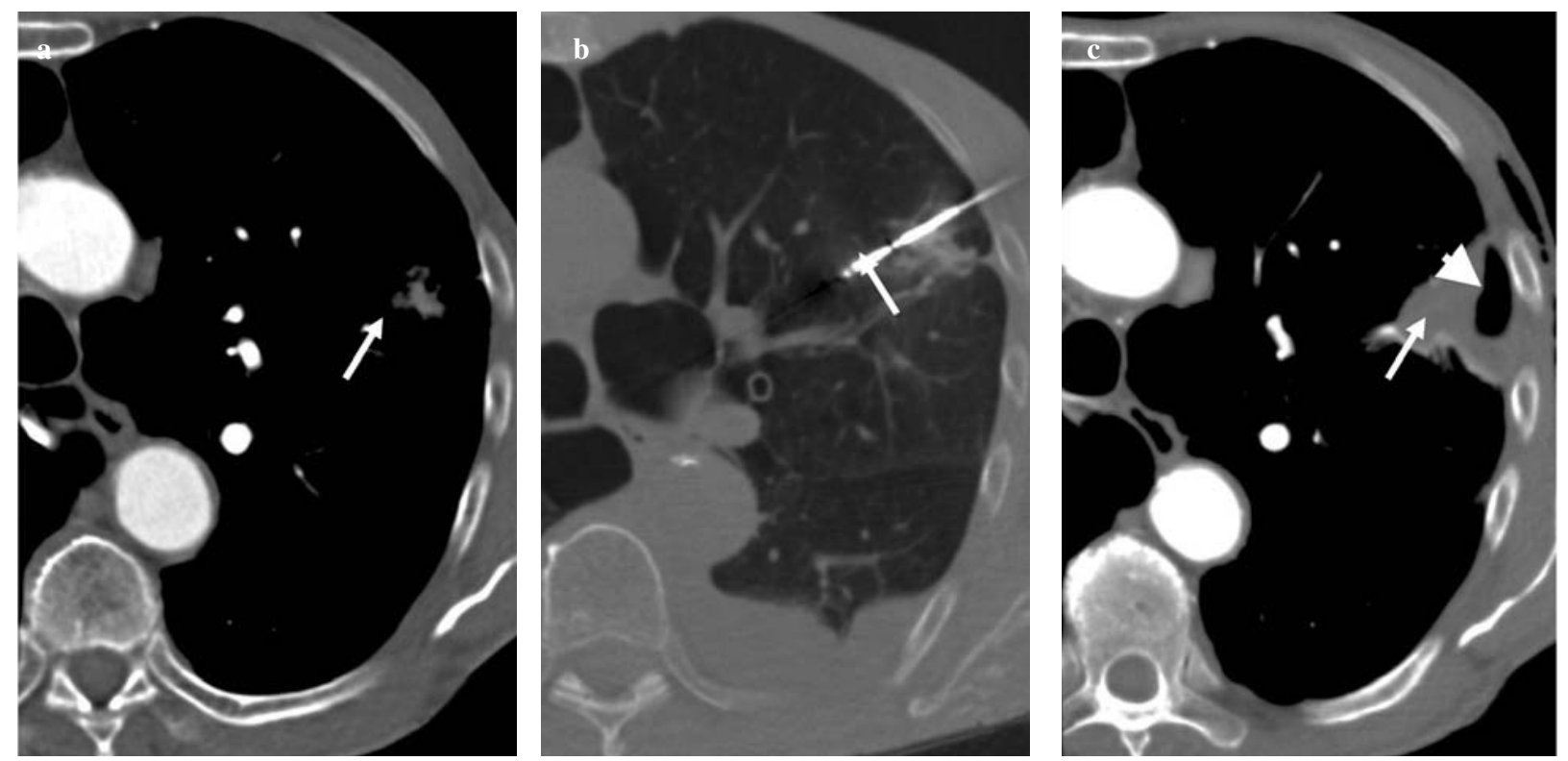

Fig. 1 a CT: solid nodular lesion (arrow) at the level of the apical segment of the left lower lobe. b CT fluoroscopy: the antenna (arrow) can be seen within the lesion. $\mathbf{c ~ C T}$ at 3 months: complete necrosis (arrow) and cavitation (arrowhead).

Fig. 1a TC: lesione nodulare solida (freccia) a livello del sergmento apicale de lobo polmonare inferiore sinistro. $\boldsymbol{b}$ Fluoro-TC: presenza dell'antenna (freccia) all'interno della lesione. c TC a 3 mesi: presenza di necrosi completa (freccia) e di cavitazione (punta di freccia).

moablation [3, 4]. The most widely used technique in clinical practice is RFA [5]. MWA is a relatively new technique that can be applied to different types of tumours and offers all the benefits of RFA as well as some substantial advantages. These include a larger volume of cellular necrosis, reduction in procedure times, greater temperatures delivered to the target lesion, the possibility of using multiple antennae simultaneously, efficacy on lesions with a cystic component and/or in proximity to vascular structures $>3 \mathrm{~mm}$ in diameter with a reduction in the heat-sink effect, and less intraprocedural pain [5, 6, 12-21]. In addition, MWA does not require placement of grounding pads [5]. In RFA, direct tissue heating is confined to an area of only a few millimetres surrounding the active electrode, whereas heating of the remaining target zone takes place through simple thermal conduction $[3,5,6,17$, $21,26]$. In contrast, microwaves induce oscillation of water molecules at a speed between 2 and 5 billion times per second, depending on the frequency of the microwaves themselves, thus producing a better convection profile and causing more uniform coagulation and necrosis of the target $[5,6,15$, $16,21]$. RFA is also limited by the increase in impedance, with boiling and charring of the tissue, due to vaporisation of water molecules that act as electrical insulators. As microwaves generate an electromagnetic wave electrically independent of the antenna, they do not seem to be subject to an increase in impedance, thanks to the nature of the waves themselves $[2,5,6,21,27,28]$. The MWA system allows simultaneous placement of multiple antennae, each connected to its own generator, thus making possible areas of necrosis of parenchima polmonare. All'imaging durante il follow-up è stata identificata la presenza di cavitazione nel 50\% dei casi (5/10 lesioni) (Fig. 1). La presenza di soffusioni pleuriche minori è stata riscontrata nel $20 \%$ dei casi $(2 / 10$ lesioni). Nel 10\% dei casi (1/10 lesioni) si è sviluppata linfoadenopatia con dimensioni massime $<1 \mathrm{~cm}$ a livello della regione della lesione trattata.

In un caso è stata osservato a 3 mesi dal trattamento la presenza di recidiva della malattia (Fig. 2). La paziente è stata quindi sottoposta ad ulteriore trattamento ablativo con microonde a 3 dal primo, determinando la completa necrosi della recidiva valutata mediante TC a pochi giorni dalla procedura. La durata media del follow-up è stata di 3,6 mesi (range 1-9 mesi), registrando in tutti i pazienti il periodo a partire dalla data della procedura fino alla scansione TC più recente.

\section{Discussione}

Nel corso degli anni sono state sviluppate diverse tecniche ablative per il controllo locale di neoplasie inoperabili: ablazione con etanolo, laserablazione, crioablazione $e$ termoablazione mediante radiofrequenze [3, 4]. Attualmente la metodica più diffusamente impiegata nella pratica clinica è la radiofrequenza [5]. Le MW sono un trattameno ablativo relativamente nuovo applicabile a differenti tipologie di neoplasie, in grado di offrire tutti $i$ benefici della radiofrequenza, presentando inoltre 

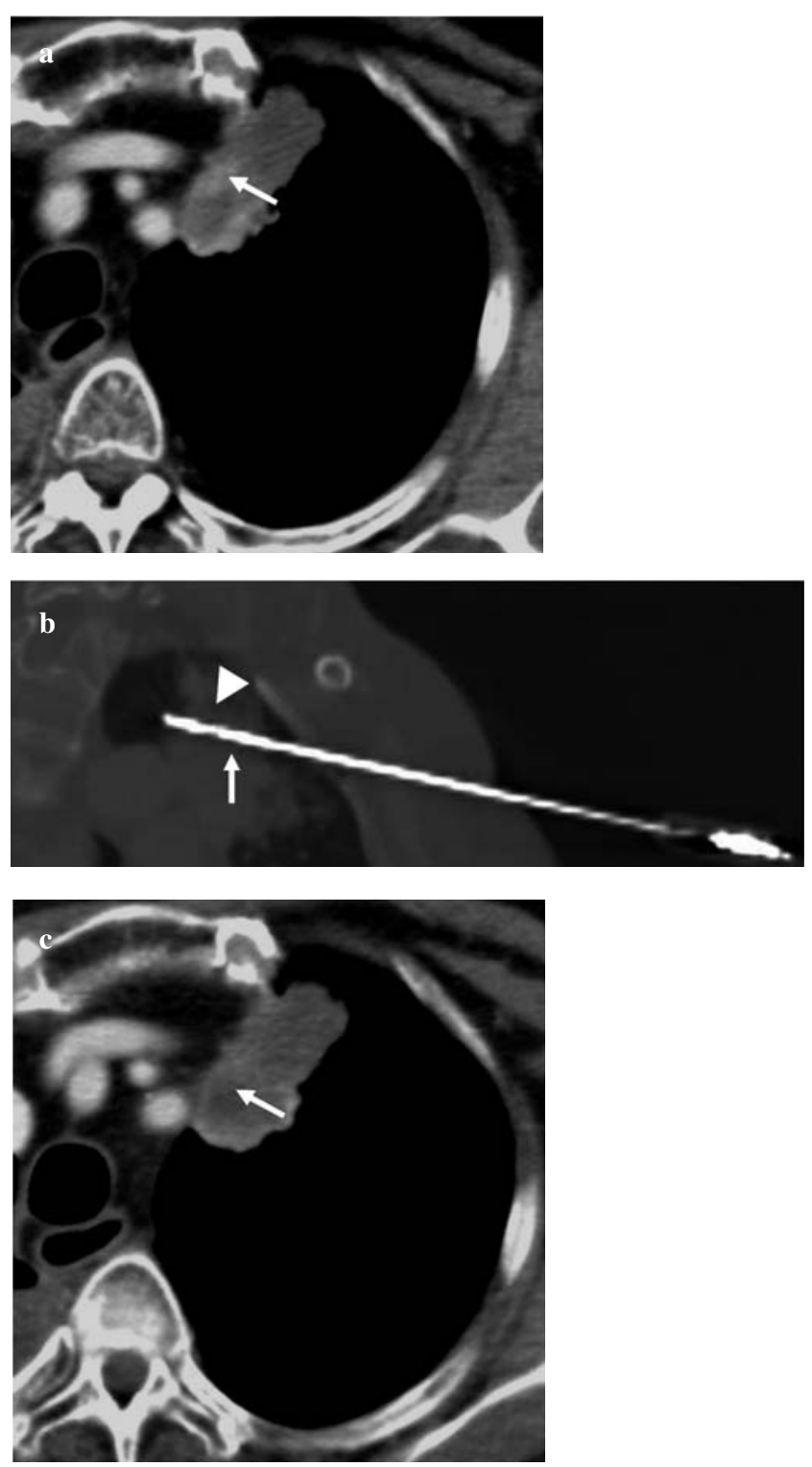

Fig. 2 a CT at 3 months from initial treatment with MWA: recurrent lesion (arrow) at the level of the apicoposterior segment of the left upper lobe. $\mathbf{b}$ CT fluoroscopy: placement of the antenna (arrow) within the lesion (arrowhead). c CT at 3 months from the second treatment with MWA: complete ablation of the lesion (arrow).

Fig. 2a TC a 3 mesi dal primo trattamento con MW: lesione recidivante (freccia) a livello del segmento apico-dorsale del lobo polmonare superiore sinistro. $\boldsymbol{b}$ Fluoro-TC: posizionamento dell'antenna (freccia) nel contesto della lesione (punta di freccia). c TC a 3 mesi dal secondo trattamento con MW: completa ablazione della lesione (freccia).

up to $6 \mathrm{~cm}$ and therefore exceeding the dimensional limitations of RFA [5, 6, 14, 21]. A comparison of the findings of our study with those reported in the literature with regard to RFA shows that the mean necrosis diameter obtained with microwaves is greater (mean diameter $3.5 \mathrm{~cm}$ ) than with RFA $(1.7 \mathrm{~cm})[3,29]$. Even the study by Brace et al. and Durich et al. $[20,21]$ shows that mean diameter and mean transverse area of necrosis are greater with MWA than with RFA: mean diameter by $25 \%$ and mean transverse area by $50 \%$ (Table 4 ). sostanziali vantaggi, quali: volumi di necrosi cellulare più ampi, riduzione del tempo di procedura, maggiori temperature alla lesione target, possibilità di utilizzare multiple antenne simultaneamente, efficacia su lesioni con componente cistica elo in prossimità di strutture vascolari $>3$ $\mathrm{mm}$ di diametro con riduzione dell'heat sink effect, minore dolore intraprocedurale [5, 6, 12-21]. Inoltre l'ablazione con $M W$ non richiede il posizionamento di grounding pads [5]. Nelle ablazioni con radiofrequenze l'attività diretta di surriscaldamento dei tessuti è limitata ad una zona che circonda l'elettrodo attivo per soli pochi millimetri, mentre l'aumento della temperatura nella restante zona target avviene attraverso semplice conduzione termica [3, 5, 6, 17, 21, 32]. Le microonde invece, inducendo l'oscillazione delle molecole d'acqua con movimenti di rotazione su se stesse ad una velocità di 2-5 miliardi di volte al secondo dipendente dalla frequenza delle onde stesse, permettono di ottenere un migliore profilo convettivo, determinando una necrosi coagulativa al target più uniforme $[5,6,15,16,21]$. Le ablazioni con radiofrequenze sono inoltre limitate dall'incremento dell'impedenza con ebollizione e carbonizzazione del tessuto, a causa dell'evaporazione delle molecole d'acqua che funge da isolante elettrico, le microonde, generando un'onda elettromagnetica elettricamente indipendente dall'antenna, non sembrano inceve essere soggette ad aumento di impedenza, grazie alla natura stessa delle onde [3, 5, 6, 21, 33, 34]. Il sistema ablativo mediante microonde consente il posizionamento simultaneo di multiple antenne, ognuna connessa al proprio generatore, al fine di ottenere aree di necrosi fino a $6 \mathrm{~cm}$, superando quindi $i$ limiti dimensionali della RFA [5, 6, 14, 21]. Comparando $i$ dati riscontrati in questo studio con $i$ dati presenti in letteratura in merito alla RFA si nota come il diametro medio di necrosi ottenuto mediante l'impiego delle microonde è maggiore (diametro medio $3,5 \mathrm{~cm}$ ) rispetto al diametro medio post-RFA (diametro medio $1,7 \mathrm{~cm}$ ) [3, 26]. Già nello studio di Brace et al. e Durick et al. [20, 21] si nota come il diametro medio e l'area trasversale media di necrosi presentino maggiori dimensioni con le microonde rispetto alla radiofrequenza: diametro medio maggiore del $25 \%$ ed area trasversale media maggiore del $50 \%$ con le microonde rispetto alla radiofrequenza (Tabella 4) [20]. Le dimensioni delle lesioni non sono risultate essere influenti sul tasso di mortalità cancrospecifica e sul tasso di sopravvivenza. Ciò risulta essere in contraddizione con studi in letteratura in merito al trattamento di NSCLC inoperabili mediante ablazione RFA [27-30]. Questo dato potrebbe derivare dalla breve durata media del follow-up (3,6 mesi). La presenza di cavitazione è statisticamente correlata alla riduzione della mortalità cancro-specifica $(p=0,02)$ [12]. Lo sviluppo di linfoadenopatia non è significativamente correlata al 
Table 4 Studies reporting microwave ablation (MWA) therapy of lung tumours

\begin{tabular}{|c|c|c|c|c|c|}
\hline \multicolumn{6}{|l|}{$\underline{\text { Clinical studies }}$} \\
\hline Author (year) & $\begin{array}{l}\text { No. patients/ } \\
\text { No. ablations }\end{array}$ & Approach & Ablation & Results & Follow-up \\
\hline Wolf et al. [12] & $50 / 66$ & Percutaneous & $\begin{array}{l}\text { 14.5-gauge } \\
\text { straight antenna }\end{array}$ & $\begin{array}{l}\text { Mortality at } 30 \text { days: } 0 \% \\
\text { Technical success: } 95 \% \\
\text { Local complications: } 9.1 \% \\
\text { Pneumothorax: } 39 \% \\
\text { Haemoptysis: } 6.6 \% \\
\text { Skin burns: } 36 \%\end{array}$ & $\begin{array}{l}\text { At } 1 \text { year: local } \\
\text { control } 67 \%\end{array}$ \\
\hline \multicolumn{6}{|c|}{ Experimental studies } \\
\hline Author (year) & $\begin{array}{l}\text { No. patients / } \\
\text { No. ablations }\end{array}$ & Approach & Ablation & Results & \\
\hline Durick et al. [21] & $\begin{array}{l}8 \text { swine/ } \\
24 \text { ablations }\end{array}$ & Thoracotomy & $\begin{array}{l}\text { 17-gauge triaxial } \\
\text { antenna }+17 \text {-gauge } \\
\text { single straight } \\
\text { antenna }\end{array}$ & \multicolumn{2}{|c|}{$\begin{array}{l}\text { Diameters of coagulation zones obtained with } \\
\text { triaxial antenna larger than diameters obtained } \\
\text { with single antenna }\end{array}$} \\
\hline Brace et al. [20] & $\begin{array}{l}3 \text { swine/18 ablations } \\
\text { (9 MWA + } 9 \text { RFA) }\end{array}$ & Percutaneous & $\begin{array}{l}\text { 17-gauge triaxial } \\
\text { (MWA) + 17-gauge } \\
\text { electrode (RFA) }\end{array}$ & \multicolumn{2}{|c|}{$\begin{array}{l}\text { Transverse diameter of necrosis } 50 \% \text { larger and } \\
\text { necrosis volume } 133 \% \text { greater than with RFA }\end{array}$} \\
\hline
\end{tabular}

$M W A$, microwave ablation; $R F A$, radiofrequency ablation

Tabella 4 Letteratura sulle microonde nel trattamento del polmone

\begin{tabular}{|c|c|c|c|c|c|}
\hline \multicolumn{6}{|l|}{ Studi clinici } \\
\hline Autore (anno) & $\begin{array}{l}\text { Numero pazienti/ } \\
\text { Numero ablazioni }\end{array}$ & Approccio & Ablazione & Risultati & Follow-up \\
\hline Wolf et al. [12] & $50 / 66$ & Percutaneo & $\begin{array}{l}14,5 \mathrm{G} \\
\text { antenna dritta }\end{array}$ & $\begin{array}{l}\text { Mortalità a } 30 \text { giorni: } 0 \% \\
\text { Successo tecnico: } 95 \% \\
\text { Complicanze locali : } 9,1 \% \\
\text { Pneumotorace: } 39 \% \\
\text { Emottisi: } 6,6 \% \\
\text { Ustioni: } 36 \%\end{array}$ & $\begin{array}{l}\text { Ad } 1 \text { anno: } \\
\text { controllo } \\
\text { locale } 67 \%\end{array}$ \\
\hline \multicolumn{6}{|l|}{ Studi sperimentali } \\
\hline Autore & $\begin{array}{l}\text { Numero pazientil } \\
\text { Numero ablazioni }\end{array}$ & Approccio & Ablazione & Risultati & \\
\hline Durick et al. [21] & $\begin{array}{l}8 \text { maiali/ } \\
24 \text { ablazioni }\end{array}$ & Toracotomico & $\begin{array}{l}17 \text { G antenna } \\
\text { triassiale }+17 G \\
\text { antenna singola } \\
\text { diritta }\end{array}$ & \multicolumn{2}{|c|}{$\begin{array}{l}\text { Diametri di necrosi coagulativa ottenuti con } \\
\text { antenna triassiale di maggiori dimensioni } \\
\text { rispetto ai diametri ottenuti con singola antenna }\end{array}$} \\
\hline Brace et al. [20] & $\begin{array}{l}3 \text { maiali/18 ablazioni } \\
(9 M W A+9 R F A)\end{array}$ & Percutaneo & $\begin{array}{l}17 \text { G antenna } \\
\text { triassiale }(M W A) \\
+17 G \text { elettrodo } \\
(R F A)\end{array}$ & \multicolumn{2}{|c|}{$\begin{array}{l}\text { Diametri trasversali di necrosi maggiori del } \\
50 \% \text {, con un volume di necrosi maggiore del } \\
133 \% \text { rispetto alla RFA }\end{array}$} \\
\hline
\end{tabular}

MWA, ablazione con microonde; RFA, ablazione con radiofrequenza 
Lesion dimension had no influence on cancer-specific mortality and survival rates. This appears to be in contradiction with previous studies reporting the treatment of unresectable NSCLC with RFA [30-33]. This could be due to the short mean duration of follow-up in our study (3.6 months). The presence of cavitation is statistically correlated with the cancer-specific mortality rate $(p=0.02)$ [12]. Lymphadenopathy was not significantly correlated with the cancer-specific and non-cancer-specific mortality rate. The presence of lymphadenopathy is related to a postablation inflammatory reaction and not to metastatic foci [11]. Evaluation of the safety of microwaves shows that the 30-day postMWA mortality rate $(0 \%)$ is markedly lower than both the post-RFA mortality rate $(3.9 \%, 6 / 153$ patients) and postsurgical mortality rate $(2.0 \%, 1 / 66$ patients) $[3,31]$. However, only in the study by Allen et al. [33] was the mortality rate dependent on treatment: death (1/66 patients) was caused by postoperative infectious complications.

In our study, asymptomatic grade 1 pneumothorax occurred in $2 / 9$ patients $(22 \%)$. This finding appears to run counter to the tendency demonstrated by Wolf et al. [12], who report a rate of $39 \%$ (26/66 patients), even greater than the post-RFA rate $(28.4 \%)$. The authors suggest that the greater dimensions of the microwave antennae with respect to the needle electrode used in RFA may favour development of pneumothorax. They reported no cases of skin burns induced by microwave-related thermal damage.

Each patient in our study was evaluated for the presence of postablation syndrome, a common and transitory post-RFA phenomenon consisting of flu-like symptoms (fever, malaise, pain, myalgia, nausea and vomiting) [14]. No patient in our study was diagnosed with postablation syndrome. It can therefore be hypothesised that MWA is less likely to cause the syndrome. Intraprocedural pain during MWA was less than during RFA, most likely due to the absence of the passage of an electrical current through the patient's body.

In conclusion, our preliminary experience supports the studies available in the literature regarding MWA, which demonstrate that the technique is safe and effective and enables local control of solid lung masses in patients who are not candidates for surgical resection $[6,12,21]$. tasso di mortalità cancro-specifica e non. La presenza di linfoadenopatia è da riferire alla reazione flogistica postablazione e non a foci metastatici [11]. Valutando la sicurezza delle microonde si osserva come il tasso di mortalità a 30 giorni post-ablazione $M W(0 \%)$ è nettamente inferiore rispetto al tasso di mortalità post-RFA (3,9\%, 6 di 153 pazienti) e post-chirurgia (2,0\%, 1 di 66 pazienti) [3, 31]. Tuttavia solo nello studio di Allen et al. il tasso di mortalità è risultato essere dipendente dal trattamento: la morte ( 1 su 66 pazienti) è stata causata da complicanze infettive post-resezione chirurgica [33].

In questo studio si è riscontrato pneumotorace in 2/9 pazienti (22\%), di grado I/asintomatico. Questo dato risulta essere in controtendenza rispetto a quanto mostrato da Wolf et al. [12]: lo studio mostra infatti un tasso del 39\% (26 su 66 pazienti), maggiore anche al tasso post-RFA $(28,4 \%)$ [12]. Wolf et al. ipotizzano che le maggiori dimensioni dell'antenna per microonde, rispetto all'agoelettrodo usato per la radiofrequenza, possano facilitare lo sviluppo di penumotorace [12]. In questo studio non si sono verificate ustioni cutanee indotte dal danno termico microonde-correlato [12].

Ciascun paziente è stato indagato in merito alla sindrome post-ablazione. La sindrome post-ablazione è un fenomeno transitorio e comune post-RFA associato a sintomatologia simil-influenzale (febbre, malessere, dolore, mialgia, nausea e vomito) [14]. In questo studio in nessun paziente è stata diagnosticata sindrome post-ablazione. È possibile quindi ipotizzare come il trattamento ablativo con microonde sia meno soggetto allo sviluppo di tale sindrome. Il dolore procedurale durante l'ablazione con microonde risulta minore rispetto al trattamento con radiofrequenze, probabilmente grazie al mancato passaggio di corrente elettrica attraverso il corpo del paziente.

In conclusione la nostra esperienza preliminare supporta gli studi in letteratura sulla termoablazione con microonde: questa tecnica risulta essere sicura, efficace, permettendo il controllo locale di neoplasie solide al polmone in pazienti non candidabili all'intervento chirurgico $[6,12,21]$.

\section{Conflict of interest None}

\section{References/Bibliografia}

1. Shugarman LR, Mack K, Sorbero ME et al (2009) Race and sex differences in the receipt of timely and appropriate lung cancer treatment. Med Care 47:774-781
2. Rossi A, Maione P, Colantuoni G et al (2009) Recent developments of targeted therapies in the treatment of non-small cell lung cancer. Curr Drug Discov Technol 6:91-102
3. Simon CJ, Dupuy DE, DiPetrillo TA et al (2007) Pulmonary radiofrequency ablation: long-term safety and efficacy in 153 patients. Radiology 243:268-275 
4. Grieco CA, Simon CJ, Mayo-Smith WW et al (2006) Percutaneous imageguided thermal ablation and radiation therapy: outcomes of combined treatment for 41 patients with inoperable stage I/II non-small-cell lung cancer. J Vasc Interv Radiology 17:1117-1124

5. Simon CJ, Dupuy DE, Mayo-Smith WW (2005) Microwave ablation: principles and applications. RadioGraphics 25:S69-S83

6. Carrafiello G, Laganà $\mathrm{D}$, Mangini $\mathrm{M}$ et al (2008) Microwave tumors ablation: principles, clinical applications and review of preliminary experiences. Int J Surg 6:65-69

7. Goetz MP, Callstrom MR, Charboneau JW et al (2004) Percutaneous imageguided radiofrequency ablation of painful metastases involving bone. A multicenter study. J Clin Oncol 22:300-306

8. Solbiati L, Livraghi T, Goldeberg SN et al (2001) Percutaneous radio-frequency ablation of hepatic metastases from colorectal cancer: long-term results in 117 patients. Radiology 221:150-166

9. Livraghi T, Goldeberg SN, Lazzaroni S et al (2000) Hepatocellular carcinoma: radiofrequency ablation of medium and large lesion. Radiology 214:1761-1768

10. Gervais DA, McGovern FJ, Arellano RS et al (2003) Renal cell carcinoma: clinical experience and technical successs with radio-frequency ablation of 41 tumors. Radiology 226:417-424

11. Cariati M, Giordano G, Midulla M et al (2007) Radiofrequency ablation of pulmonary lesions. Radiology Med 112:149-156

12. Wolf FJ, Grand DJ, Machan JT et al (2008) Microwave ablation of lung malignancies: effectiveness, CT findings, and safety in 50 patients. Radiology 247:871-879

13. Wright SA, Sampson LA, Warner TF et al (2005) Radiofrequency versus microwave ablation in a hepatic porcine model. Radiology 236:132-139

14. Carrafiello $G$, Laganà $D$, Ianniello A et al (2007) Post-radiofrequency ablation syndrome after percutaneous radiofrequency of abdominal tumours: one centre experience and review of published works. Australas Radiology 51:550-554
15. Fen W, Liu W, Li C et al (2002) Percutaneous microwave coagulation therapy for lung cancer. Zhounghua Zhong Liu Za Zhi 24:388-390

16. Wright SA, Lee FT, Mahvi DM (2003) Hepatic microwave ablation with multiple antennae results in synergistically larger zones of coagulation necrosis. Ann Surg Oncol 10:275-283

17. Skinner MG, Iizuka MN, Kolios MC et al (1998) A theoretical comparison of energy sources-microwave, ultrasound and laser-for interstitial thermal therapy. Phys Med Biol 43:3535-3547

18. Shock SA, Meredith K, Warner TF (2004) Microwave ablation with loop antenna: in vivo porcine liver model. Radiology 231:143-149

19. Stauffer PR, Rossetto F, Prakash M et al (2003) Phantom and animal tissues for modelling the electrical properties of human liver. Int J Hyperthermia 19:89-101

20. Brace CL,Hinshaw JL, Laeseke PF et al (2009) Pulmonary thermal ablation: comparison of radiofrequency and microwave devices by using gross pathologic and CT findings in a swine model. Radiology 251:705-711

21. Durick NA, Laeseke PF, Broderick LS et al (2008) Microwave ablation with triaxial antennas tuned for lung: results in an in vivo porcine model

22. Sacks D, McClenny TE, Cardella JF et al (2003) Society of Interventional Radiology Clinical Practice Guidelines. J Vasc Interv Radiology 14:199-202

23. Hollen PJ, Gralla RJ, Kris MG et al (2005) A comparison of visual analogue and numerical rating scale formats for the Lung Cancer Symptom Scale (LCSS): does format affect patient ratings of symptoms and quality of life quality of life? Qual Life Res 14:837-847

24. Kaplan EL, Meier P (1958) Nonparametric estimation from incomplete observations. J Am Stat Assoc 53:457-481
25. de Baere T, Palussiere J, Auperin A et al (2006) Midterm local efficacy and survival after radiofrequency ablation of lung tumors with minimum followup of 1 year: prospective evaluation. Radiology 240:587-596

26. Goldberg SN, Stein MC, Gazelle GS et al (1999) Percutaneous radiofrequency tissue ablation: optimization of pulsedradiofrequency technique to increase coagulation necrosis. J Vasc Interv Radiology 10:907-916

27. Lu DS, Raman SS, Limanond $P$ et al (2003) Influence of large peritumoral vessels on outcome of radiofrequency ablation of liver tumors. J Vasc Interv Radiology 14:1267-1274

28. Oshima F, Yamakado K, Akeboshi M et al (2004) Lung radiofrequency ablation with and without bronchial occlusion: experimental study in porcine lungs. Vasc Interv Radiology 15:1451-1456

29. Gazelle GS, Goldberg SN, Solbiati L et al (2000) Tumor ablation with radiofrequency energy. Radiology 217:633-646

30. Jin GY, Lee JM, Lee YC et al (2004) Primary and secondary lung malignancies treated with percutaneous radiofrequency ablation: evaluation with follow-up helical CT. AJR Am J Roentgenol 183:1013-1020

31. Belfiore G, Moggio G, Tedeschi E et al (2004) CT-guided radiofrequency ablation: a potential complementary therapy for patients with unresectable primary lung cancer-a preliminary report of 33 patients. AJR Am J Roentgenol 183:1003-1011

32. Yasui K, Kanazawa S, Sano Y et al (2004) Thoracic tumors treated with CT-guided radiofrequency ablation: initial experience. Radiology 231:850-857

33. Allen MS, Darling GE, Pechet TT et al (2006) Morbidity and mortality of major pulmonary resections in patients with early-stage lung cancer: initial results of randomized, prospective trial. Ann Thorac Surg 81:1013-1020 